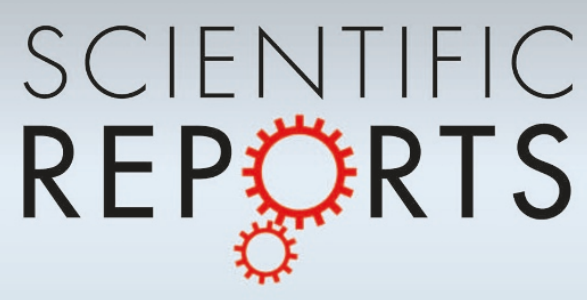

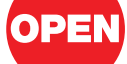

SUBJECT AREAS:

ELECTRONIC PROPERTIES AND DEVICES

COMPUTATIONAL SCIENCE FLUID DYNAMICS

ELECTRONIC PROPERTIES AND MATERIALS

Received

10 October 2012

Accepted

5 December 2012

Published

11 January 2013

Correspondence and requests for materials should be addressed to M.M. (millmen@gmail.

com)

\section{Hydrodynamic Model for Conductivity in Graphene}

\author{
M. Mendoza' ${ }^{1}$, H. J. Herrmann' \& S. Succi ${ }^{2,3}$
}

'ETH Zürich, Computational Physics for Engineering Materials, Institute for Building Materials, Schafmattstrasse 6, HIF, CH-8093 Zürich (Switzerland), ${ }^{2}$ Istituto per le Applicazioni del Calcolo C.N.R., Via dei Taurini, 1900185 , Rome (ltaly), ${ }^{3}$ Freiburg Institute for Advanced Studies, Albertstrasse, 19, D-79104, Freiburg, Germany.

Based on the recently developed picture of an electronic ideal relativistic fluid at the Dirac point, we present an analytical model for the conductivity in graphene that is able to describe the linear dependence on the carrier density and the existence of a minimum conductivity. The model treats impurities as submerged rigid obstacles, forming a disordered medium through which graphene electrons flow, in close analogy with classical fluid dynamics. To describe the minimum conductivity, we take into account the additional carrier density induced by the impurities in the sample. The model, which predicts the conductivity as a function of the impurity fraction of the sample, is supported by extensive simulations for different values of $\varepsilon$, the dimensionless strength of the electric field, and provides excellent agreement with experimental data.

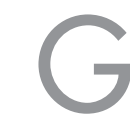

raphene has revealed a series of amazing properties, such as ultra-high electrical conductivity ${ }^{1,2}$, ultra-low shear viscosity to entropy ratio $^{3}$, as well as exceptional structural strength, as combined with mechanical flexibility ${ }^{4}$ and optical transparency ${ }^{5}$. Many of these fascinating properties are due to the fact that, consisting of literally one single carbon monolayer, graphene represents the first instance ever of a truly twodimensional material (the "ultimate flatland"6). Moreover, due to the special symmetries of the honeycomb lattice, electrons in graphene are shown to behave like an effective Dirac fluid of massless chiral quasi-particles, propagating at a Fermi speed of about $c \sim 10^{6} \mathrm{~m} / \mathrm{s}^{3,7}$. This configures graphene as an unique, slow-relativistic electronic fluid, where many unexpected quantum-electrodynamic phenomena can take place ${ }^{8}$. For instance, since electrons are about 300 times slower than photons, their mutual interaction is proportionately enhanced, leading to an effective fine-structure constant $\alpha_{g r}=e^{2} / \hbar v_{F} \sim 1$. As a result of such strong interactions, it has been recently proposed that this peculiar $2 \mathrm{D}$ graphene electron gas should be characterized by an exceptionally low viscosity/entropy ratio (near-perfect fluid), coming close to the famous AdS-CFT lower bound conjectured for quantum-chromodynamic fluids, such as quark-gluon plasmas ${ }^{8}$. This spawns the exciting prospect of observing electronic pre-turbulence in graphene samples, as first pointed out in Ref.3 and confirmed by recent numerical simulations?

Some of the electrical properties of graphene are still not fully understood, such as the linear increase of the electrical conductivity with the number of charge carriers, the existence of a minimum conductivity (see Ref. 10, and reference therein), and even the nature of the main scattering mechanism limiting the carrier mobility ${ }^{11}$. In fact, classical transport theories, based on short-range scattering of electrons by impurities, predict that the electric conductivity in graphene should be independent of the carrier density ${ }^{12}$. Recent works in the field ${ }^{13,14}$ have shown that such linear dependence might be potentially explained by treating the impurities as screened Coulomb scatterers. Nevertheless, some measurements of the change in the electrical conductivity upon immersion of graphene samples in high- $\kappa$ dielectric media differ from this conclusion ${ }^{15,16}$. Here, we construct a model for describing the electrical conductivity in graphene by using a completely different approach, which is based on the recently developed picture of an electronic ideal relativistic fluid at the Dirac point. We demonstrate that, although this model is based on a semiclassical theory (it cannot take into account all quantum effects, e.g. Landau quantization, quantum hall effects, and quantum interference), it captures the main factors that contribute to such linear behavior and the appearance of a minimum conductivity.

Since the most likely relevant limiting factor for the graphene conductivity is still subject of controversy, e.g. it can be due to random charged impurity centers ${ }^{13}$ or strong neutral defects that induce resonant scattering ${ }^{15,16}$, we will treat the impurities as hard-spheres, hindering the electron flow (scattering electrons), similarly to the way a disordered medium does in the context of fluid dynamics. The choice of hard-spheres is based on the experimental results by Monteverde et al. ${ }^{11}$, which suggested that electrons seem to collide mostly with short range scatterers of the size of a few carbon-carbon interatomic distances, like voids, adatoms, etc. Since the relativistic 
fluid approach is derived from the quantum Boltzmann equation (QBE) for graphene ${ }^{17}$, a hydrodynamic description of the conductivity can be expected to apply as long as the QBE collision operator takes proper account of the Coulomb interactions between electrons. Therefore, once Coulomb interactions are included in the viscosity of the fluid, the conductivity (which in our case, unlike viscosity, is a property of the flow rather than of the fluid) becomes a function of the dissipation introduced in the system by the impurities, i.e. the electron-impurity interaction.

Here, we treat graphene as a disordered medium and develop a model for its conductivity, as a function of the impurity density describing the anomalous dependence of the conductivity on the carrier density and the minimum conductivity due to the carrier density induced by the impurities. The results are compared with experimental data yielding very satisfactory agreement.

\section{Results}

Electronic fluid in graphene. Our treatment is based on the hydrodynamic equations derived by Müller et al. ${ }^{3,7}$, based on the quantum Boltzmann equation for electrons in graphene. This analysis delivers the value of the transport coefficients, namely the fluid shear viscosity, which is an input parameter in our model. The hydrodynamic approach in graphene is valid when the inelasticscattering rate due to electron-electron interactions dominates. This is the case at low doping, at high temperatures, and in moderate fields ${ }^{18}$. However, to neglect electron-phonon interactions, we will have to stay at a moderately high temperature of around $100 \mathrm{~K}^{19}$. In absence of magnetic fields, the quasiparticle distribution function, $f_{s}$, evolves according to the quantum Boltzmann equation,

$$
\frac{\partial f_{s}}{\partial t}+\vec{v}_{s} \cdot \nabla f_{s}+e \vec{E} \cdot \nabla_{\vec{k}} f_{s}=-\Omega\left[f_{s}\right]
$$

where $\vec{E}$ is an external electric field, $e$ the electric charge of the electron, $\Omega\left[f_{s}\right]$ a collision operator that takes into account the electron-electron interactions, $\vec{v}_{s}=s c \vec{k} /|\vec{k}|, \vec{k}$ the wave vector, $c$ the Fermi speed $\left(\sim 10^{6} \mathrm{~m} / \mathrm{s}\right)$, and the sign $s$, not to be confused with the entropy density, distinguishes between electrons $(+)$ and holes $(-)^{18,20}$. At equilibrium, the probability distribution function becomes the Fermi-Dirac distribution,

$$
f_{s}(t, \vec{x}, \vec{k})=\frac{1}{e^{(s c|\vec{k}|-\mu) / k_{B} T}+1},
$$

where $\mu$ is the chemical potential and $T$ denotes the temperature. Thus, in the hydrodynamic limit, from Eqs. (1) and (2) one can derive the equations for the Dirac electron fluid in graphene: $\partial \rho / \partial t+\nabla \cdot(\rho \vec{u})=0$, for charge conservation; $\partial \epsilon / \partial t+\nabla \cdot[(\epsilon+p) \vec{u}]$ $=0$, for energy density conservation and

$$
\rho_{r}\left[\frac{\partial \vec{u}}{\partial t}+(\vec{u} \cdot \nabla) \vec{u}\right]+\nabla p+\frac{\vec{u}}{c^{2}} \frac{\partial p}{\partial t}-\eta \nabla^{2} \vec{u}=\rho \vec{E},
$$

for momentum conservation. Here, $\epsilon$ is the energy density, $p$ the pressure, $\rho$ the charge density, $\vec{u}$ the drift velocity, $\rho_{r} \equiv(\epsilon+p) / c^{2}$, and $\eta$ the shear viscosity.

For the case of undoped graphene $(\mu=0)$, the presence of charge density is due to the thermal energy and can be described by,

$$
\rho=\rho_{\text {th }}=e\left(\frac{k_{B} T}{\hbar c}\right)^{2} .
$$

However, when there are impurities, they can induce electric potentials on the graphene sample and a correction due to the chemical potential must be considered,

$$
\rho=\rho_{t h} \Phi_{\rho}\left(\mu / k_{B} T\right),
$$

where $\Phi_{\rho}$ is a dimensionless increasing function defined in Ref. 7. Note that, in our analytical model, we will use this concept in order to introduce a minimum conductivity in the graphene sample, where the function $\Phi_{\rho}$ will be modeled by a free parameter to fit the experimental data and will take into account not only the carriers generated by the impurities but also other kind of phenomena that could contribute to induce carrier density.

The shear viscosity $\eta$, in Eq. (3), can be calculated using

$$
\eta=C_{\eta} \frac{M\left(k_{B} T\right)^{2}}{4 \hbar c^{2} \alpha^{2}}
$$

where $C_{\eta} \sim O(1)$ is a numerical coefficient, $\alpha=e^{2} / \mathcal{E} \hbar c$ is the effective fine structure constant, $\mathcal{E}$ the relative dielectric constant of the substrate, and $M$ the number of species of free massless Dirac particles ${ }^{3,7}$. Additionally, the entropy densities can be calculated according to the Gibbs-Duhem relation $\epsilon+p=T s$. These equations have been derived under the assumption $|\vec{u}|<c$, and therefore the relativistic correction term, $\propto \partial p / \partial t$, can be neglected, so that the classical Navier-Stokes equations are recovered. Note that, despite the high speed of the electrons, $|\vec{u}| \sim 0.1 c$, the Reynolds number remains moderate, due to nano-metric size of the samples and the high kinematic viscosity of the electronic fluid in graphene.

Kinematic viscosity. Based on Ref. 3, the dynamic viscosity of graphene in a sample of linear size $L_{0}$, is given by Eq. (6). This equation can be written in the following form:

$$
\eta=C_{\eta} \frac{M}{4 \alpha^{2}}\left(\frac{k_{B} T}{\hbar \omega_{f}}\right)^{2} \frac{\hbar}{L_{0}^{2}},
$$

where we have introduced the characteristic frequency $\omega_{f}=c / L_{0}$, and by solving the appropriate quantum Boltzmann equation, it is concluded that $C_{\eta} \simeq 0.449$. Eq. (7) can also be rewritten as $\eta=C_{\eta} q_{f}^{-2} \hbar / L_{0}^{2}$, where $q_{f} \equiv \hbar \omega_{f}\left(k_{B} T\right)$. Note that, in order for a classical (non quantum) picture of electron fluid to apply, the energy of excitations must be much lower than the thermal energy, i.e. $q_{f} \ll 1$, the so-called collision-dominated regime. Taking a typical set of parameters (in MKS units), $c=10^{6}, L_{0}=10^{-6}, T=100 \mathrm{~K}$, and $\eta / s \sim 0.2 \hbar / k_{B}$, we obtain $\eta \sim 10^{-20}$. Since the Reynolds number is dictated by the kinematic viscosity of the fluid, $v$, rather than by the dynamic one, $\eta=\rho v$, with no need of involving the mass density, it is of interest to estimate the kinematic viscosity of the electron fluid in graphene.

To this purpose, we appeal to the definition of the Reynolds number as given in Ref. 3, namely:

$$
R e=\frac{s / k_{B}}{\eta / \hbar} \frac{k_{B} T}{\hbar \omega_{f}} \frac{u_{0} L_{0}}{v_{0}},
$$

where $v_{0}=c L_{0}$. By writing $R e=u_{0} L_{0} / v$ and equating with the above, we obtain

$$
v=v_{0} \frac{\hbar \omega_{f}}{k_{B} T} \frac{s / k_{B}}{\eta / s} .
$$

Using $\eta / s=0.2 \hbar / k_{B}{ }^{3}$ and $q_{f} \simeq 0.07$, we obtain $v \simeq 10^{-2}$. To be noted that, in spite of its extremely low dynamic viscosity, the kinematic viscosity of graphene is about four orders of magnitude larger than that of water. These four orders of magnitude are more than compensated by the large speed of the electrons, which is ultimately responsible for the sizeable values of the Reynolds numbers which can be achieved in graphene samples at micron scales. For instance, by taking $u_{0}=0.1 c \sim 10^{5} \mathrm{~m} / \mathrm{s}$, for a sample of 1 micron in length, we obtain $R e \sim 20$ for the global sample, and about $R e \sim 0.04$ on the scale of the impurities.

Analytical model description. In this work, we will treat impurities as circular rigid obstacles of diameter $d$. This choice is not arbitrary, but it is based on the fact that some experiments ${ }^{11,15,16}$ suggest the the main scattering mechanism in graphene is due to strong neutral 
defects, with a range shorter than the Fermi wavelength, inducing resonant scattering. Thus, the diameter $d$ can be interpreted as the characteristic length for the range of the interaction electronimpurity.

Let us now assume that the electronic fluid moves in the $x$ direction as a consequence of an applied electric field $E$, and $\nabla \cdot\left(\rho_{r} \vec{u}\right) \simeq 0$ (incompressible limit). Therefore, we can calculate the force $\vec{F}$ acting on a single impurity due to the electronic flow, as $\vec{F}=\oint \overleftrightarrow{\Pi} \cdot d \vec{l}$, where $\overleftrightarrow{\Pi}$ is the stress tensor defined by $\Pi_{i j}=p \delta_{i j}+\rho_{r} u_{i} u_{j}+\pi_{i j}$, with the viscous tensor $\pi_{i j}=\eta\left(\partial u_{i} / \partial x_{j}+\partial u_{j} / \partial x_{i}\right)$, and $\vec{l}$ is a unit vector perpendicular to the impurity circle. Here the indices $i, j=1,2$ denote the coordinates $x$ and $y$. Thus, solving the equations for the Dirac electron fluid, in the steady state (all time derivatives are neglected), and following an analogous procedure as in classical hydrodynamics ${ }^{21}$, we obtain for the drag force, $F_{D}=F_{x}$,

$$
F_{D}=\lambda \eta v,
$$

where $\lambda$ is a dimensionless parameter that depends on the Reynolds number. Here, $v$ is the velocity of the fluid very far from the impurity. We first simulated single impurities with different sizes and different fluid velocities (see Fig. 1), obtaining that a linear approximation is justified in the range of relevance to this work. Note that, for a perfect fluid $(v=0)$, there is no drag force. However, from the point of view of the kinematic viscosity, electrons in graphene are far from being a perfect fluid. Therefore, we expect them to play a crucial effect on the drag force controlling the total conductivity of the sample.

Let us denote by $\phi$ the impurity fraction, namely the ratio between the area not occupied by the impurities and the total area of the system, $\phi=1-N \pi d^{2} /(4 A)$, with $N$ the number of impurities in a sample of area $A$. We can thus propose the relation between $v$ and the total current density as $J=\phi \rho v$. Note that $\phi \rho$ is the existent electronic charge density in the graphene sample, since the volume fraction $1-$ $\phi$ is occupied by the impurities.

Let us consider a representative elementary area of the sample of length $d_{x}$ in the direction of the flow and $d_{y}$ across it. With $\mathcal{N}$ circular impurities in this area, we can write $\mathcal{N}=4(1-\phi) d_{x} d_{y} /\left(\pi d^{2}\right)$. The total force acting on the electronic fluid due to impurities (equal to the total force acting on the impurities due to the fluid), is given by $F_{\text {tot }}=\mathcal{N} F_{D}$. Here, the distance between impurities is taken sufficiently large enough to prevent the flow close to an impurity from affecting the flow nearby another impurity.

In order to describe correctly the physics of graphene, we need to include in our model an extra feature. Due to the linear Dirac-Weyl spectrum of graphene, and the non-existence of a gap between the conduction and valence bands, the slightest amount of impurities or an external potential will induce charge carriers in the graphene sample ${ }^{22,23}$, see Eq. (5). Thus, in our model, the total amount of carriers induced by the impurities will be proportional to the impurity concentration, $(1-\phi) A$, leading to an extra carrier density in the fraction of the sample occupied by the electronic fluid, $\phi A$.The extra carriers are then given by $\gamma(1-\phi) / \gamma$, where $\gamma$ is the proportionality constant that characterizes the impurity-electron interaction.

Summing the forces, namely the Lorentz and drag forces, acting on the elementary area leads to: $\phi \rho E d_{x} d_{y}+\gamma(1-\phi) E d_{x} d_{y} / \phi-F_{t o t}=0$, and by inserting the value of $F_{t o t}$, we obtain

$$
F_{D}=\frac{\rho \pi d^{2}}{4}\left(\frac{\phi}{1-\phi}+\frac{\gamma}{\rho \phi}\right) E .
$$

Replacing Eq. (10), taking into account that $J=\phi \rho v$ and Ohm's law, we can identify the conductivity as:

$$
\sigma=\frac{\rho^{2} \pi d^{2}}{4 \eta \lambda}\left(\frac{\phi^{2}}{1-\phi}+\frac{\gamma}{\rho}\right)=\sigma_{0} \frac{\phi^{2}}{1-\phi}+\sigma_{\text {min }},
$$

where we have introduced the coefficients $\sigma_{0}=\rho^{2} \pi d^{2} /(4 \eta \lambda)$, and $\sigma_{\text {min }}=\sigma_{0} \gamma / \rho$. This equation represents the key result of our paper.

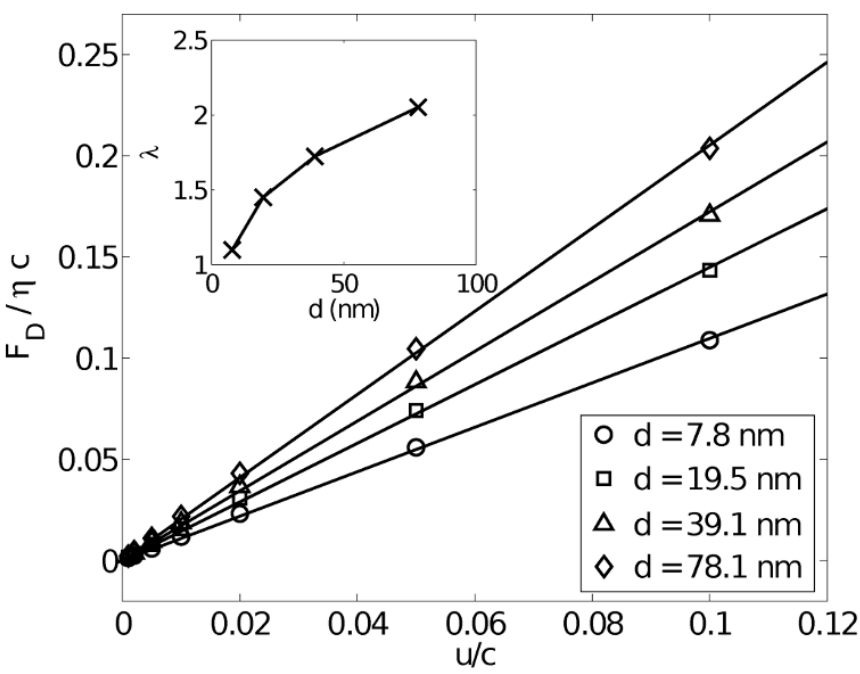

Figure $1 \mid$ Drag force acting on a single impurity. Drag force $F_{D}$ acting on a single impurity as a function of the graphene flow drift velocity for different impurity diameters. The solid lines represent the linear dependency of the drag force with the velocity of the fluid. In the inset, the dependence of the dimensionless parameter $\lambda$ on the impurity diameter is shown.

An analogous derivation, for fluid dynamics in disordered media, can be found in Ref. 24, 25. Note that $\sigma_{0}$ also can be written as $\sigma_{0}=$ $n\left(e^{2} \pi d^{2} c^{2} / 12 k_{B} T \nu \lambda\right)$, where $n=\rho / e$ is the electronic number density and $v$ the kinematic viscosity. From this expression, we can see that the conductivity in graphene depends linearly on the carrier density, thus implying a constant mobility $\mu=\sigma / n e$, in agreement with experimental observations ${ }^{1,26}$. In addition, our model can also explain why the mobility remains almost constant in the range of temperatures where $v \propto T^{-1}$ (see Ref. 9), and the presence of a minimum conductivity in graphene, second term on the rhs of Eq. (12), $\sigma_{\text {min }}=e \pi d^{2} c^{2} \gamma / 12 k_{B} T \nu \lambda$, which is independent of the carrier and impurity densities. Indeed, this model cannot explain, as other theoretical models, the sublinear behavior of the conductivity for the zero range impurity because, in that range, point defects and boundary conditions start to be dominant. In addition, it cannot describe the electron-phonon interaction either, since these have been excluded at the outset. All features above will make the object of future extensions of this work.

Dimensionless numbers. For the numerical validation, and in order to obtain general results, we will use dimensionless numbers. For this purpose, we can rewrite Eq. (3) alternatively as

$$
\frac{\partial \vec{u}}{\partial t}+(\vec{u} \cdot \nabla) \vec{u}+\frac{1}{\rho_{r}} \nabla p+\frac{\vec{u}}{\rho_{r} c^{2}} \frac{\partial p}{\partial t}-\frac{\eta}{\rho_{r}} \nabla^{2} \vec{u}=\frac{\rho}{\rho_{r}} \vec{E},
$$

and therefore we obtain,

$$
\frac{\partial \vec{u}}{\partial t}+(\vec{u} \cdot \nabla) \vec{u}+\frac{1}{\rho_{r}} \nabla p+\frac{\vec{u}}{\rho_{r} c^{2}} \frac{\partial p}{\partial t}-v \nabla^{2} \vec{u}=\frac{\rho}{\rho_{r}} \vec{E},
$$

where $v$ represents the kinematic viscosity. Let us define the following relations: $\vec{u}=u_{0} \vec{u}^{\prime}, \vec{t}=t_{0} \vec{t}^{\prime},(x, y, z)=L_{0}\left(x^{\prime}, y^{\prime}, z^{\prime}\right), \rho_{r}=\rho_{r 0} \rho_{r}{ }^{\prime}, \rho=$ $\rho_{0} \rho^{\prime}$, and $\vec{E}=E_{0} \vec{E}^{\prime}$, where the prime quantities are dimensionless, and $u_{0}, t_{0}, L_{0}, \rho_{r 0}, \rho_{0}$, and $E_{0}$ are characteristic values for the respective physical quantities. Thus, using the state equation $\epsilon=2 p$, we can deduce $p=\frac{1}{3} \rho_{r 0} u_{0}^{2} p^{\prime}$ and $\epsilon=\frac{2}{3} \rho_{r 0} u_{0}^{2} \epsilon^{\prime}$. Replacing these relations in Eq. (14), multiplying by $\frac{t_{0}}{u_{0}}$, and using the relation $u_{0}=\frac{L_{0}}{t_{0}}$, we obtain, 

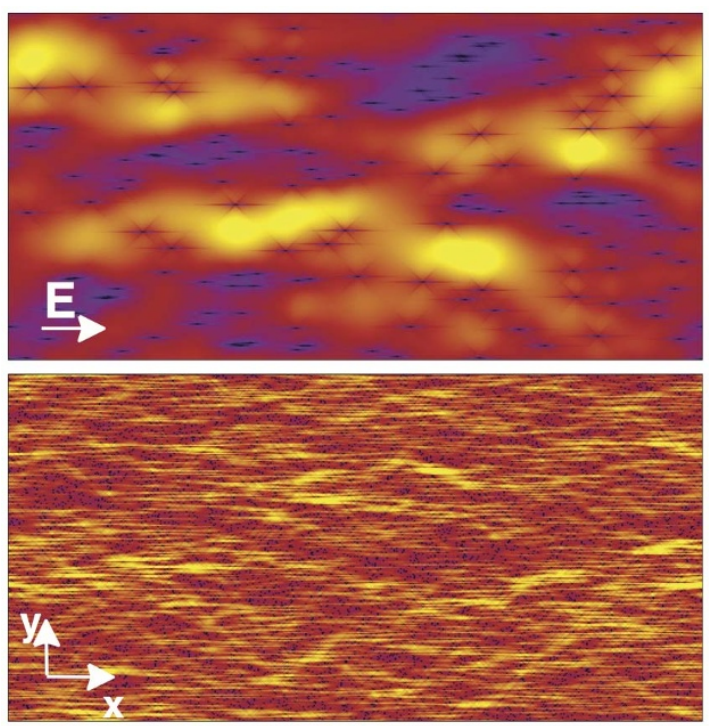

Figure 2 Speed of the electronic flow. Absolute value of the velocity in graphene with multiple impurities, for two different impurity fractions, 0.952 (bottom) and 0.999 (top). The electric field is applied in the $x$ direction (from left to right) and set up to $1.77 \mathrm{~V} / \mathrm{m}$.

$$
\frac{\partial \vec{u}^{\prime}}{\partial t^{\prime}}+\left(\vec{u}^{\prime} \cdot \nabla^{\prime}\right) \vec{u}^{\prime}+\frac{1}{3 \rho_{r}^{\prime}} \nabla^{\prime} p^{\prime}+\frac{1}{3} \frac{\mu_{0}^{2^{\prime}}}{c^{2}} \vec{u}^{\prime} \frac{\partial p^{\prime}}{\partial t^{\prime}}-\frac{v}{\mu_{0} L_{0}} \nabla^{\prime 2} \vec{u}^{\prime}=\frac{\rho_{0} E_{0} L_{0}}{\rho_{r 0} \mu_{0}^{2}} \frac{\rho^{\prime}}{\rho_{r}^{\prime}}
$$

To simplify this equation, we can equal the characteristic velocity to the Fermi speed, $u_{0}=c$. Therefore, we obtain

$$
\frac{\partial \vec{u}^{\prime}}{\partial t^{\prime}}+\left(\vec{u}^{\prime} \cdot \nabla^{\prime}\right) \vec{u}^{\prime}+\frac{1}{3 \rho_{r}^{\prime}} \nabla^{\prime} p^{\prime}+\frac{\vec{u}^{\prime}}{3} \frac{\partial p^{\prime}}{\partial t^{\prime}}-\frac{v}{\mu_{0} L_{0}} \nabla^{\prime 2} \vec{u}^{\prime}=\frac{\rho_{0} E_{0} L_{0}}{\rho_{r 0} \mu_{0}^{2}} \frac{\rho^{\prime}}{\rho_{r}^{\prime}}
$$

We can identify two characteristic dimensionless numbers. The first one is the Reynolds number, which is, $R e=\frac{u_{0} L_{0}}{v}$, and the second one, which we call " $\mathcal{E}$ number" is defined by $\mathcal{E}=\frac{\rho_{0} E_{0} L_{0}}{\rho_{r 0} u_{0}^{2}}=\frac{\rho_{0} V_{0}}{\rho_{r 0} u_{0}^{2}}$, where $V_{0}=E_{0} L_{0}$ is the characteristic electrical potential. Using these definitions, we get

$$
\frac{\partial \vec{u}^{\prime}}{\partial t^{\prime}}+\left(\vec{u}^{\prime} \cdot \nabla^{\prime}\right) \vec{u}^{\prime}+\frac{1}{3 \rho_{r}^{\prime}} \nabla^{\prime} p^{\prime}+\frac{\vec{u}^{\prime}}{3} \frac{\partial p^{\prime}}{\partial t^{\prime}}-\frac{1}{R e} \nabla^{\prime 2} \vec{u}^{\prime}=\mathcal{E} \frac{p^{\prime}}{p_{r}^{\prime}} \vec{E}^{\prime}
$$

Note that this equation is dimensionless and therefore the universal features of the dynamics of the system are controlled only by the numbers $R e$ and $\mathcal{E}$ : the latter measures the strength of the electric drive, while the former scales inversely with the dissipation opposing this drive. For notational simplicity, we will remove primes, leading to

$$
\frac{\partial \vec{u}}{\partial t}+(\vec{u} \cdot \nabla) \vec{u}+\frac{1}{3 \rho_{r}} \nabla p+\frac{\vec{u}}{3} \frac{\partial p}{\partial t}-\frac{1}{R e} \nabla^{2} \vec{u}=\mathcal{E} \frac{\rho}{\rho_{r}} \vec{E},
$$

Numerical results. Fig. 2 illustrates the speed of the fluid for two different impurity densities, dark and yellow colors denoting low and high speeds respectively. An electric field of $1.77 \mathrm{~V} / \mathrm{m}$ was applied in $x$ direction (from left to right). Here we can see that for high impurity fraction (see Fig. 2, top), the speed of the fluid presents fluctuations on larger scales affecting the total conductivity of the sample. From the calculation of the electric current density and the electric field, we obtain the Ohm's law, giving a slope $\sigma$.

The conductivity $\sigma$ is calculated from the numerical slopes and plotted as a function of the impurity fraction. The inset of Fig. 3 reports the comparison between the analytical solution, using Eqs. (12), showing an excellent agreement with the numerical data.

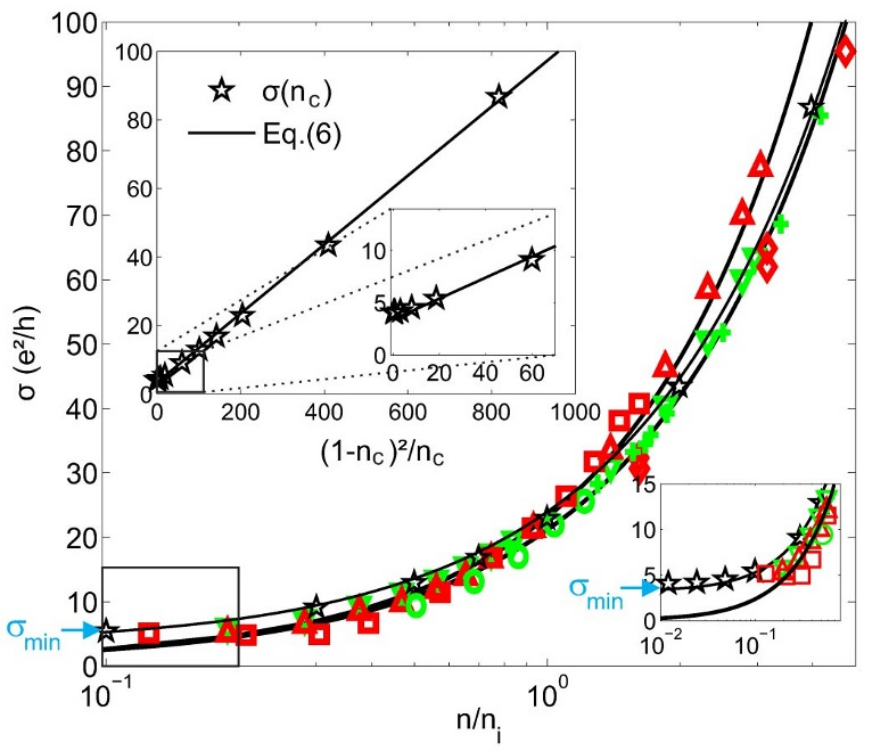

Figure $3 \mid$ Comparison between our analytical model and experimental data. Comparison between our results (stars) and experimental data for the conductivity $\sigma$, as a function of $n / n_{i}$. Data from Ref. 1 are represented by up and down triangles, from Ref. 23 by circles and squares, and from Ref. 31 by diamonds and crosses, for electrons and holes respectively. Solid lines from bottom to top, theory for separations $\delta=0$ according to Ref. 13, our results, and theory for $\delta=0.2 \mathrm{~nm}$ according to the previous reference. In the inset (top), we show the conductivity as a function of $\phi^{2} /(1-\phi)$, with an inset to observe the minimum conductivity. In the inset (bottom) we amplify the region close to the Dirac point.

For the fitting parameters, we obtain $\sigma_{0}=(9.9 \pm 0.1) \times 10^{-2} e^{2} / h$, and $\sigma_{\min }=3.4 \pm 0.6$. Note that there is a difference between our analytical model and the numerical simulations for the minimum conductivity. This difference is due to the fact that, for high impurity densities, the flow around one impurity starts to affect the flow around the others, and therefore, Eq. (12) needs some additional terms. In particular, the approximation $F_{\text {tot }}=\mathcal{N} F_{D}$ does not hold anymore and non-linear correction terms should be included. Thus, while the minimum conductivity given by the analytical model is $\sim$ $3.4 e^{2} / h$, the simulations give $\sim 4 e^{2} / h$. We have verified that the conductivity of graphene, as computed in our model, does not show any appreciable dependence on the size of the system.

In order to compare with experiments, we express the conductivity in terms of the ratio $n / n_{i}$, where $n_{i}=\left(2.91 \times 10^{16} \mathrm{~m}^{-2}\right)(1-\phi)$, in our case. According to this expression and setting $n=n_{0}$, we obtain that $\xi \equiv(1 / \alpha) n / n_{i}=(1-\phi)^{-1}$, with $\alpha=4.85 \times 10^{-3}$. Inserting this result into Eq. (12), we obtain $\sigma / \sigma_{0}=\xi(1-1 / \xi)^{2}$. Note that for values $\xi \gg$ 1, i.e. $n / n_{i} \gg \alpha=4.85 \times 10^{-3}$, this equation tends to

$$
\sigma \simeq \sigma_{0} \xi+\sigma_{\min }=\frac{\sigma_{0}}{\alpha} \frac{n}{n_{i}}+\sigma_{\min }
$$

This corresponds to the linear dependence obtained by different theoretical models for graphene $\mathrm{e}^{13,14,22,23,27-30}$.

In Fig. 3, we see the dependence between the conductivity and the ratio $n / n_{i}$, and we clearly observe the prediction for the minimum conductivity of our model. The experimental data have been taken from Refs. 1, 23, 31, and compared with the results of the present work, showing good agreement. In Fig. 3, we also compare with the model proposed by Hwang et al. ${ }^{13}$, where the impurities are located in a plane (substrate) parallel to the layer of graphene, with a separation $\delta$ between the layers. In Fig. 4 , we compare our results with Coulomb impurity charges in random phase approximation (RPA $)^{13}$. In the RPA model, the Boltzmann transport equation is used with impurities that are located randomly in the graphene sample. Our model 
shows good agreement in the slope with the RPA model, however, we achieve higher values due to the shift made by the minimum conductivity.

\section{Discussion}

We have developed an analytical model which accounts for a linear behavior of the conductivity with the electron density $n$, as well as with the ratio $n / n_{i}$, in the limit $n / n_{i} \gg 4.5 \times 10^{-3}$. In addition, it can also model the minimum conductivity in graphene as a consequence of the carrier density induced by the presence of impurities. Our model is based on a hydrodynamic description of electron flow in graphene, whereby Coulomb interactions are included through the viscosity of the electron fluid, and is valid in the collision-dominated regime. In this model, the impurities are treated as hard-sphere obstacles submerged on the electronic fluid, based on the fact that some experiments ${ }^{11,15,16}$ suggest that strong short-range neutral scatterers are the main scattering mechanism in graphene. Although this idea and the one about the longrange Coulomb scatterers are still object of controversy, the fact that the present analytical model can account for the conductivity of graphene suggests that indeed the short-range scattering models might be appropriate for graphene.

This work is based on the hydrodynamic description of electrons in graphene proposed in Ref. 3, 7, which is a model developed for undoped graphene that neglects the electron-impurity and electronphonon interactions. Here we have -extended- this approach by adding the electron-impurity interactions through a macroscopic porous media approach. Since this approach rests on basic conservation laws, it is supposedly very robust and independent on the validity of an underlying quantum Boltzmann equation, so long the microscopic interactions justify the build-up of a macroscopic viscosity (no superconductivity or other macroscopic quantum effects of that sort). Thus, our model is able to reproduce experimental results to a satisfactory degree of accuracy.

For the set of parameters investigated in the present work, linear Ohm's law appears to apply throughout. However, based on Ref. 9, by increasing the size of the impurities (less screening), non-Ohmic behavior could occur, due the onset of pre-turbulent phenomena within the graphene sample. It would be very interesting to verify such possibility by future experiments, as well as the inclusion of the electron-phonon interaction to model both, suspended and supported samples, at higher temperatures.

\section{Methods}

For the simulation, we use the hydrokinetic fluid solver proposed by Mendoza et al. ${ }^{32-34}$, adapted to two-dimensional flow in graphene'. The simulation was implemented on a grid of size $256 \times 512$ cells, representing a rectangular graphene sample of size $1.5 \times 3 \mu \mathrm{m}$. We set up samples with a fixed number of impurities located randomly on the grid, each impurity covering one cell size, varying $\phi$ between 0.4 and 0.999 . The Dirac-quasiparticle fluid in graphene has a kinematic viscosity $v=$ $8.57 \times 10^{-3} \mathrm{~m}^{2} / \mathrm{s}$ (see Ref. 9), and by taking the Fermi speed $u_{0}=10^{6} \mathrm{~m} / \mathrm{s}$ as a characteristic speed, we obtain a Reynolds number $R e=350$. Equating $R e$ for both systems, in physical and numerical units, the cell size and time step are fixed to $\delta x=$ $5.86 \mathrm{~nm}$ and $\delta t=5.86 \mathrm{fs}$. For a given temperature, $T_{0}=100 \mathrm{~K}$ in our case, we can calculate the carrier density induced by the thermal energy with Eq. (4), $n_{0}=1.411 \times$ $10^{14} \mathrm{~m}^{-2}$ and therefore, using the approximate relation $\epsilon=2 n_{0} k_{B} T_{0}^{3,7}$, the energy density $\epsilon=3.90 \times 10^{-7} \mathrm{~J} / \mathrm{m}^{2}$ and the density $\rho_{r 0}=5.84 \times 10^{-19} \mathrm{~kg} / \mathrm{m}^{2}$. Using the electron charge, we obtain the charge density, $\rho_{0}=2.26 \times 10^{-5} \mathrm{C} / \mathrm{m}^{2}$. In numerical units, these values correspond to $n_{0}=4.845 \times 10^{-3}, \epsilon=2 / 3$, and $\rho_{r 0}=\rho_{0}=1.0$, where the charge of the electron is $e=2.064 \times 10^{2}$. Using the characteristic velocity $u_{0}$, we can calculate the value of the characteristic current density $J_{0}=\rho_{0} u_{0}=22.6 \mathrm{~A} /$ $\mathrm{m}$ or $J_{0}=\rho_{0} u_{0}=1.0$ in physical and numerical units, respectively. On the other hand, to obtain realistic values of $\mathcal{E}$, we use a characteristic electric field of $E_{0}=4.41 \mathrm{~V} / \mathrm{m}$, which in numerical units corresponds to $E_{0}=10^{-6}$. In this work, $\mathcal{E}$ takes values from $10^{-5}$ to $2 \times 10^{-4}$.

To model the extra carrier density induced by the impurities, as described in Eq. (5), we introduce an extra density charge $\Delta \rho$ localized on each impurity position. Therefore, each impurity contributes a quantity $\rho^{*}=\Delta \rho d x^{2} / A$ to the total charge of the sample, such that $\rho=\rho_{t h}+\rho^{*} N=\rho_{t h}+\Delta \rho n_{i}$, where $n_{i}$, denotes the impurity density. This linear dependence between $\rho$ and $n_{i}$ is in qualitative agreement with experimental data ${ }^{23}$. We made several simulations for different values of $\Delta \rho$, finding

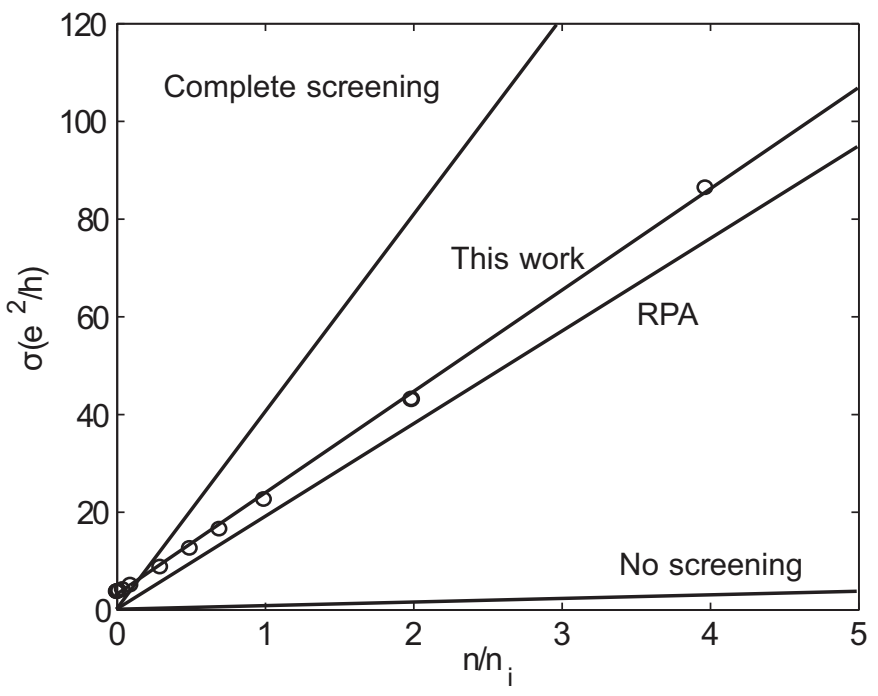

Figure $4 \mid$ Comparison between analytical approaches. Conductivity $\sigma$ as a function of $n / n_{i}$ for different types of scattering models ${ }^{13}$. RPA is the conductivity calculated by using a random phase approximation with Coulomb scatterers. The unscreened Coulomb interaction would yield a conductivity smaller than the minimum value in graphene, over the entire range of gate voltages.

that $\Delta \rho=60$ leads to a minimum conductivity of $4 e^{2} / h$. In our analytical model, this value corresponds to $\gamma=60$, in numerical units. The simulations ran up to $5 \times 10^{5}$ time steps.

1. Novoselov, K. et al. Two-dimensional gas of massless dirac fermions in graphene. Nature Letters 438, 197 (2005).

2. Novoselov, K. S. et al. Electric Field Effect in Atomically Thin Carbon Films. Science 306, 666-669 (2004).

3. Müller, M., Schmalian, J. \& Fritz, L. Graphene: A nearly perfect fluid. Phys. Rev. Lett. 103, 025301 (2009).

4. Lee, C., Wei, X., Kysar, J. W. \& Hone, J. Measurement of the elastic properties and intrinsic strength of monolayer graphene. Science 321, 385-388 (2008).

5. Kuzmenko, A. B., van Heumen, E., Carbone, F. \& van der Marel, D. Universal optical conductance of graphite. Phys. Rev. Lett. 100, 117401 (2008).

6. Geim, A. K. \& MacDonald, A. H. Graphene: Exploring carbon flatland. Phys. Today 35 (2007).

7. Müller, M. \& Sachdev, S. Collective cyclotron motion of the relativistic plasma in graphene. Phys. Rev. B 78, 115419 (2008).

8. Shuryak, E. Why does the quark-gluon plasma at rhic behave as a nearly ideal fluid? Progress in Particle and Nuclear Physics 53, 273-303 (2004).

9. Mendoza, M., Herrmann, H. J. \& Succi, S. Preturbulent regimes in graphene flow. Phys. Rev. Lett. 106, 156601 (2011).

10. Das Sarma, S., Adam, S., Hwang, E. H. \& Rossi, E. Electronic transport in twodimensional graphene. Rev. Mod. Phys. 83, 407-470 (2011).

11. Monteverde, M. et al. Transport and elastic scattering times as probes of the nature of impurity scattering in single-layer and bilayer graphene. Phys. Rev. Lett. 104, 126801 (2010).

12. Shon, N. H. \& Ando, T. Quantum transport in two-dimensional graphite system. Journal of the Physical Society of Japan 67, 2421-2429 (1998).

13. Hwang, E. H., Adam, S. \& Das Sarma, S. Carrier transport in two-dimensional graphene layers. Phys. Rev. Lett. 98, 186806 (2007).

14. Nomura, K. \& MacDonald, A. H. Quantum transport of massless dirac fermions. Phys. Rev. Lett. 98, 076602 (2007).

15. Jang, C. et al. Tuning the effective fine structure constant in graphene: Opposing effects of dielectric screening on short- and long-range potential scattering. Phys. Rev. Lett. 101, 146805 (2008).

16. Ponomarenko, L. A. et al. Effect of a high- $\kappa$ environment on charge carrier mobility in graphene. Phys. Rev. Lett. 102, 206603 (2009).

17. Fritz, L., Schmalian, J., Muller, M. \& Sachdev, S. Quantum critical transport in clean graphene. Phys. Rev. B 78, 085416 (2008).

18. Müller, M., Fritz, L. \& Sachdev, S. Quantum-critical relativistic magnetotransport in graphene. Phys. Rev. B 78, 115406 (2008).

19. Bao, W. S., Liu, S. Y., Lei, X. L. \& Wang, C. M. Nonlinear dc transport in graphene. Journal of Physics: Condensed Matter 21, 305302 (2009).

20. Mendoza, M., Araújo, N. A. M., Succi, S. \& Herrmann, H. J. Transition in the equilibrium distribution function of relativistic particles. Sci. Rep. 2, 611 (2012).

21. Oseen, C. Über die stokes'sche formel, und über eine verwandte aufgabe in der hydrodynamik. Arkiv för matematik, astronomi och fysik vi (1910). 
22. Adam, S., Hwang, E. H., Galitski, V. M. \& Das Sarma, S. A self-consistent theory for graphene transport. Proceedings of the National Academy of Sciences 104, 18392-18397 (2007)

23. Chen, J.-H. et al. Charged-impurity scattering in graphene. Nat. Phys. Lett. 4, 377-381 (2008).

24. Rumer, R. \& Drinker, P. Resistance to laminar flow through porous media. Proc. Am. Soc. Civil Eng., J. Hydraulic Div. 92, 155 (1966).

25. Bear, J. Dynamics of Fluids in Porous Media (American Elsevier Publising Company, 1972).

26. Zhang, Y., Tan, Y.-W., Stormer, H. L. \& Kim, P. Experimental observation of the quantum hall effect and berry's phase in graphene. Nature 438, 201-204 (2005).

27. Ando, T. Screening effect and impurity scattering in monolayer graphene. Journal of the Physical Society of Japan 75, 074716 (2006).

28. Cheianov, V. V. \& Fal'ko, V. I. Friedel oscillations, impurity scattering, and temperature dependence of resistivity in graphene. Phys. Rev. Lett. 97, 226801 (2006).

29. Trushin, M. \& Schliemann, J. Minimum electrical and thermal conductivity of graphene: A quasiclassical approach. Phys. Rev. Lett. 99, 216602 (2007).

30. Yan, X.-Z., Romiah, Y. \& Ting, C. S. Electric transport theory of dirac fermions in graphene. Phys. Rev. B 77, 125409 (2008).

31. Tan, Y.-W. et al. Measurement of scattering rate and minimum conductivity in graphene. Phys. Rev. Lett. 99, 246803 (2007).

32. Mendoza, M., Boghosian, B. M., Herrmann, H. J. \& Succi, S. Fast lattice boltzmann solver for relativistic hydrodynamics. Phys. Rev. Lett. 105, 014502 (2010).
33. Mendoza, M., Boghosian, B. M., Herrmann, H. J. \& Succi, S. Derivation of the lattice boltzmann model for relativistic hydrodynamics. Phys. Rev. D 82, 105008 (2010).

34. Hupp, D., Mendoza, M., Bouras, I., Succi, S. \& Herrmann, H. J. Relativistic lattice boltzmann method for quark-gluon plasma simulations. Phys. Rev. D 84, 125015 (2011).

\section{Author contributions}

All authors conceived and designed the research, analyzed the data, worked out the theory, and wrote the manuscript.

\section{Additional information}

Competing financial interests: The authors declare no competing financial interests. License: This work is licensed under a Creative Commons Attribution-NonCommercial-NoDerivs 3.0 Unported License. To view a copy of this license, visit http://creativecommons.org/licenses/by-nc-nd/3.0/

How to cite this article: Mendoza, M., Herrmann, H.J., \& Succi, S. Hydrodynamic Model for Conductivity in Graphene. Sci. Rep. 3, 1052; DOI:10.1038/srep01052 (2013). 